\title{
COVID-19 and Thyroid Diseases: How the Pandemic Situation Affects Thyroid Disease Patients
}

\author{
Laurentius Aswin Pramono
}

Department of Public Health and Nutrition, School of Medicine and Health Sciences, Atma Jaya Catholic University of Indonesia, Jakarta, Indonesia Department of Internal Medicine, Saint Carolus Hospital, Jakarta, Indonesia

\begin{abstract}
Patients with thyroid diseases need special attention during this COVID-19 pandemic. There is a paucity of publications that review the effect of coronavirus infection on thyroid disease patients, such as those with hyperthyroidism, hypothyroidism, thyroid nodules and cancer. This article aims to collect reviews and statements about how the COVID-19 pandemic has affected the management of thyroid disease patients.
\end{abstract}

Key words: COVID-19, thyroid disease, hyperthyroidism, hypothyroidism, thyroid cancer

\section{INTRODUCTION}

The World Health Organization (WHO) announced the global pandemic situation caused by severe acute respiratory syndrome coronavirus 2 (SARS-CoV-2) as coronavirus disease 2019 (COVID-19) in March 2020. ${ }^{1}$ The disease has spread widely all over the world, including Southeast Asian countries. ${ }^{1}$ The pandemic is a great burden to all communities and populations. Older citizens and people with chronic and degenerative diseases are prone to severe illness if infected by the virus. ${ }^{2}$ Furthermore, morbidity and mortality caused by COVID-19 are higher in patients with chronic, metabolic and degenerative diseases such as diabetes, hypertension, cardiovascular disease, cancer, stroke and autoimmune diseases. ${ }^{3}$

As COVID-19 is a new illness, its effects on patients with thyroid disease are not yet known. ${ }^{4}$ Because of the prevalence of thyroid disease- 1 to $2 \%$ for spontaneous hypothyroidism, 0.5 to $2 \%$ for hyperthyroidism in women (10 times more common than in men), and $1 \%$ and $5 \%$ for clinically detectable thyroid nodules in men and women, respectively-it may be surmised that patients with the disease may be affected directly and indirectly by the pandemic situation. ${ }^{5}$ This review aims to collect publications which discuss consequences of COVID-19 in thyroid disease patients.

\section{PATHOBIOLOGY}

There are a limited number of publications on the effect of viral infections on thyroid pathology. A study on severe acute respiratory syndrome (SARS) patients during the outbreak in 2003 found that triiodothyronine (T3) and thyroxine (T4) levels were lower in SARS patients compared to controls, during the acute and convalescent phases. ${ }^{6}$
Autopsy findings in patients with SARS showed destruction of follicular and parafollicular thyroid cells which led to low T3 and T4. ${ }^{7}$ Viral infections can affect thyroid hormone synthesis causing low T4 and T3, a common phenomenon known as non-thyroidal illness syndrome.

Chronic viral infections can later lead to autoimmune disease, including thyroid diseases, such as Graves' disease and Hashimoto's thyroiditis. Viral infections account for major environmental factors in subacute and chronic autoimmune thyroiditis. ${ }^{8}$ Coxsackie virus, echovirus, EpsteinBarr virus, herpes simplex virus, mumps and parvovirus are thought to cause autoimmune thyroiditis. Presently, there are no published studies on the effect of COVID-19 and the risk for later autoimmune thyroid disease.

\section{STATEMENTS FROM THYROID SOCIETIES}

Various thyroid interest groups, including the American Thyroid Association (ATA), the European Thyroid Association (ETA), the British Thyroid Foundation (BTF) and the American Association for Clinical Endocrinologists (AACE), have released statements in their respective official websites for the guidance of thyroid patients during the COVID-19 pandemic. ${ }^{4,-11}$ The statements provide information for physicians and patients on how to deal with specific thyroid concerns during the pandemic.

Thyroid disorders may be generally divided to three major conditions, namely, hyperthyroidism, hypothyroidism, and thyroid nodules and cancer. Hyperthyroidism encompasses conditions caused by overactive thyroid function, resulting in elevated levels of thyroid hormones (free T4 and free T3) and low levels of thyroid stimulating hormone (TSH). This includes Graves' disease, toxic multinodular goiter (Plummer's disease) and toxic nodular 
goiter (toxic adenoma). Hypothyroidism is a condition of low free T4 and free T3 levels, and high TSH, as seen in csongenital hypothyroidism, endemic goiter (iodine deficiency disorder), and chronic autoimmune thyroid disease (Hashimoto's thyroiditis). Thyroid nodules and cancer are in the same category of neoplasia. The treatment of thyroid cancer includes surgery (thyroidectomy), radioactive iodine therapy (radioablation), and TSH suppression therapy with levothyroxine.

\section{HYPERTHYROIDISM}

The ATA, BTF and AACE have stated that autoimmune thyroid disease which resulted to hyperthyroidism (Graves' disease, multinodular toxic goiter or toxic adenoma) does not increase the risk for COVID-19 infection, morbidity or mortality. ${ }^{9-11}$ Patients maintained on anti-thyroid drugs or levothyroxine also do not have a higher risk for COVID-19 infection. It is important to continue thyroid medications since being untreated or suboptimally controlled may increase the risk of viral infection or complications. ${ }^{4,11}$

Treatment with anti-thyroid drugs (ATD) like propylthiouracil, carbimazole, methimazole may cause agranulocytosis, a very rare adverse reaction with symptoms resembling COVID-19. These include fever, sore throat and muscle pain., ${ }^{9,11}$ Physician assessment must be done promptly to ascertain the cause of the symptoms.

ATDs must be continued with the same dose and titration, as was done prior to the pandemic. The medications may be prescribed for two to three months to minimize the need for frequent clinic visits. ${ }^{9-11}$ Maintaining euthyroidism as long as possible can prevent more severe conditions such as thyroid storm, an unexpected severe complication of viral infections in uncontrolled hyperthyroid patients. ${ }^{12}$

In Graves' orbitopathy or other forms of thyroid-related eye disease, the use of high dose $(500 \mathrm{mg})$ intravenous glucocorticoid (methylprednisolone) weekly for six weeks may suppress the immune system and increase the risk for infection, including COVID-19; or worsen blood glucose control which can lead to prolonged length of stay in the hospital. ${ }^{11}$ The decision to give this therapy has to be made after having fully discussed the risks and benefits with the patient. The patient should be well advised to observe and maintain physical hygiene and distancing while on high dose corticosteroid therapy.

\section{HYPOTHYROIDISM}

The various etiologies of hypothyroidism such as Hashimoto's thyroiditis, post-thyroidectomy for thyroid nodule or thyroid cancer, post-radioactive iodine therapy or congenital hypothyroidism, are all treated with levothyroxine to maintain normal thyroid hormone levels. Hashimoto's thyroiditis is a chronic autoimmune condition that may present with other autoimmune conditions. The BTF reassures patients that the condition does not increase the risk of COVID-19 infection, morbidity or mortality. ${ }^{4}$

Levothyroxine must be continued with the same dose as before the pandemic., ${ }^{4-11}$ Once the patient has attained euthyroidism (normal free T4 and TSH levels) with a known total dose in a day or a week, the medication may be prescribed for 3 or 4 months to limit clinic visits. Calcium and vitamin $\mathrm{D}$ must also be continued with the same dose.

\section{THYROID NODULE AND CANCER}

The urgency to perform fine needle aspiration (FNA) biopsy or thyroid cyst aspiration is determined by the patient's risk factors, the characteristics of the nodule and clinical judgment. ${ }^{9}$ Generally, it is safe to perform FNA biopsy or cytology if standard personal protective equipment (PPE) are worn. ${ }^{13}$ If the nodule is highly suspicious for malignancy, has indicators of thyrotoxicosis, or with concomitant compression signs and symptoms, prompt referral to a head and neck, thyroid or oncologic surgeon to prepare for thyroidectomy is recommended. If the nodule is deemed moderately to highly suspicious and would be aided by preoperative cytology, FNA biopsy may be performed with a moderate level of PPE. If the nodule has very low to low suspicion of malignancy, biopsy may be postponed and the nodule may be observed by repeating thyroid ultrasonography at 3 to 6 months.

Thyroid cancer patients generally do not have a higher risk for COVID-19 infection. Majority of thyroid cancers are well-differentiated, specifically papillary and follicular thyroid cancer. ${ }^{4,9,10}$ Both types are slow growing, rarely aggressive, and infrequently metastasize to distant organs. Some well-differentiated cancers with aggressive character and higher stages from vascular invasion, lymph node metastases and distant spread pose a higher risk for viral infection including COVID-19. Morbidity and mortality can also increase, especially in those with lung metastases. ${ }^{11}$

Poorly-differentiated thyroid cancers, specifically medullary and anaplastic types, have a higher risk for morbidity and mortality from COVID-19 infection. These patients, and also those with aggressive variants of well-differentiated thyroid cancer, sometimes receive tyrosine kinase inhibitors (sorafenib, vandetanib or lenvatinib) after total thyroidectomy or radioactive iodine therapy. These agents suppress the immune system and may confer a higher risk of severe pneumonia in outbreak situations. ${ }^{4,9-11}$ Patients who have previously received external beam radiotherapy to the neck also have a higher risk for severe illness from COVID-19. ${ }^{4}$ All high risk thyroid cancer patients must then be advised to self-isolate and stay at home during the pandemic.

The decision to proceed with thyroid surgery during the pandemic is based on consideration of the surgeon, the patient's condition, the hospital (i.e., availability of operating room), the availability of personal protective equipment (PPE) and adequate screening prior to surgery. Hospitals in Jakarta, such as the Saint Carolus Hospital, perform examinations including chest $X$-ray, chest computerized tomography scan, complete blood count, C-reactive protein, lactate dehydrogenase, rapid antibody test for IgM/IgG SARS-CoV2, real time-polymerase chain reaction for SARS-CoV2 of nose and throat specimens, and adequate preoperative consultation and screening with an internal medicine specialist or pulmonologist and an anesthesiologist. ${ }^{14}$ With the application of a complete general preoperative assessment including specific screening to prevent the spread of COVID-19, thyroid surgical procedures can be performed safely. 
Table 1. Conditions that warrant urgent ( $<4$ weeks) thyroid surgery

1. Thyroid cancer which is life-threatening (large size), with local invasion to the trachea or recurrent laryngeal nerve, with aggressive features (rapid growing, adherent to nearby organs, with distant metastases)

2. Graves' disease or toxic adenoma with severe or life-threatening symptoms, which cannot be controlled by anti-thyroid medications

3. Goiter or enlargement of the thyroid gland with respiratory or gastrointestinal tract compressive symptoms

4. Open core-biopsy (and removal, total or near-total thyroidectomy) for nodules highly suspicious for thyroid cancer, such as medullary thyroid cancer (high calcitonin and with highly suspicious ultrasonographic characteristics), anaplastic thyroid cancer or thyroid lymphoma if other diagnostic modalities are equivocal or inconclusive

5. Pregnant patient with thyrotoxic and compressive symptoms presenting a life-threatening condition for the mother and fetus and cannot be controlled with anti-thyroid medications

Adapted from the American Thyroid Association. Novel coronavirus (COVID-19) and the thyroid. https://www.thyroid.org. ${ }^{9}$

Other issue is about radioactive iodine therapy during the COVID-19 pandemic. The ATA clearly states that radioactive iodine (RAI) therapy for patients with possible residual thyroid tissue after total thyroidectomy can be delayed for six months and is still effective. ${ }^{9}$ Moreover, RAI therapy does not increase the risk, morbidity and mortality of COVID-19. ${ }^{4,9,11}$ It should be noted that RAI therapy is highly dependent on the availability of adequate healthcare facilities. If the indication for RAI therapy is Graves' hyperthyroidism or toxic multinodular goiter, antithyroid medications may be preferred, as these provide an easier and simpler means of achieving euthyroidism.

\section{CONCLUSION}

During this time of the COVID-19 pandemic, thyroid disease patients must receive optimal therapy for each of their conditions, such as hyperthyroidism, thyroid eye disease, hypothyroidism, thyroid nodules and thyroid cancer. Statements from the American Thyroid Association, the European Thyroid Association, the British Thyroid Foundation and the American Association for Clinical Endocrinologists (AACE) can guide clinicians, physicians, endocrinologists and thyroid surgeons in their therapeutic decisions.

\section{Acknowledgments}

The author expresses his gratitude to the Department of Public Health and Nutrition, School of Medicine and Health Sciences, Atma Jaya Catholic University of Indonesia; the Department of Internal Medicine, Saint Carolus Hospital; and the Saint Carolus COVID-19 Outbreak Team for supporting the faculty in their teaching and academic activities, in clinical work, and in their endeavors in writing studies and reviews during the time of the COVID-19 pandemic.

\section{Statement of Authorship}

The author certifies fulfillment of ICMJE authorship criteria.

\section{Author Disclosure}

The author declared no conflicts of interest.

\section{Funding Source}

None.

\section{References}

1. Ge $\mathrm{H}$, Wang $\mathrm{X}$, Yuan $\mathrm{X}$, et al. The epidemiology and clinical information about COVID-19. Eur J Clin Microbiol Infect Dis. 2020;39(6):1011-9. PMID: 32291542. PMCID: PMC7154215. https://doi. org/10.1007/s10096-020-03874-z.

2. Liu K, Chen Y, Lin R, Han K. Clinical features of COVID-19 in elderly patients: A comparison with young and middle-aged patients. J Infect. 2020;80(6):e14-8. PMID: 32171866. PMCID: PMC7102640. https://doi. org/10.1016/j.jinf.2020.03.005.

3. Yang J, Zheng $Y$, Gou X, et al. Prevalence of comorbidities and it effects in patients infected with SARS-CoV-2: A systematic review and meta-analysis. Int J Infect Dis. 2020;94:91-5. PMID: 32173574. PMCID: PMC7194638. https://doi.org/10.1016/j.iijid.2020.03.017.

4. British Thyroid Foundation. Thyroid disease and coronavirus (COVID-19). 4 May 2020. https://www.btf-thyroid.org/news/thyroiddisease-and-coronavirus-covid-19.

5. Vanderpump MPJ. Epidemiology of thyroid disorders. In: Luster M, Duntas L, Wartofsky L, eds. The Thyroid and Its Diseases: A Comprehensive Guide for the Clinician. Cham, Switzerland: Springer, 2019.

6. Pal R, Banerjee M. COVID-19 and the endocrine system: Exploring the unexplored. J Endocrinol Invest. 2020;43(7):1027-31. PMID: 32361826. PMCID: PMC7195612. https://doi.org/10.1007/s40618-020-01276-8.

7. Wei L, Sun S, Xu C, et al. Pathology of the thyroid in severe acute respiratory syndrome. Hum Pathol. 2007;38(1):95-102. PMID: 16996569 PMCID: PMC7112059. https://doi.org/10.1016/j.humpath.2006.06.011.

8. Desailloud R, Hober D. Viruses and thyroiditis: An update. Virol J. 2009;6(5). https://doi.org/10.1186/1743-422X-6-5.

9. American Thyroid Association. Novel coronavirus (COVID-19) and the thyroid: Resources. https://www.thyroid.org/covid-19/.

10. European Thyroid Association. COVID-19: Information and recommendations for patients with thyroid diseases. ETA Public Health Board Statement. https://www.eurothyroid.com/news/covid19-thyroid-diseases.html.

11. American Association of Clinical Endocrinologists. AACE position statement: Coronavirus (COVID-19) and people with thyroid disease https://www.aace.com/recent-news-and-updates/aace-positionstatement-coronavirus-covid-19-and-people-thyroid-disease.

12. Baharoon SA. H1N1 infection-induced thyroid storm. Ann Thorac Med. 2010;5(2):110-2. PMID: 20582177. PMCID: PMC2883193. https://doi.org/10.4103/1817-1737.62475.

13. Vigliar E, Iaccarino A, Bruzzese D, et al. Cytology in the time of coronavirus disease (COVID-19): An Italian perspective. J Clin Pathol. 2020; jclinpath-2020-206614. PMID: 32312717. PMCID: PMC7211103. https://doi.org/10.1136/jclinpath-2020-206614.

14. Hardi F, Shinto R, Putra AC, Fahmi M, Wiyono WH, Pramono LA, et al. Coronavirus disease (Covid-19) service in Saint Carolus Hospital. Updated 29 April 2020. Saint Carolus Hospital, Jakarta, Indonesia.

Authors are required to accomplish, sign and submit scanned copies of the JAFES Author Form consisting of: (1) Authorship Certification, that authors contributed substantially to the work, that the manuscript has been read and approved by all authors, and that the requirements for authorship have been met by each author; (2) the Author Declaration, that the article represents original material that is not being considered for publication or has not been published or accepted for publication elsewhere, that the article does not infringe or violate any copyrights or intellectual property rights, and that no references have been made to predatory/ suspected predatory journals; (3) the Author Contribution Disclosure, which lists the specific contributions of authors; and (4) the Author Publishing Agreement which retains author copyright, grants publishing and distribution rights to JAFES, and allows JAFES to apply and enforce an Attribution-Non-Commercial Creative Commons user license. Authors are also required to accomplish, sign, and submit the signed ICMJE form for Disclosure of Potential Conflicts of Interest. For original articles, authors are required to submit a scanned copy of the Ethics Review Approval of their research as well as registration in trial registries as appropriate. For manuscripts reporting data from studies involving animals, authors are required to submit a scanned copy of the Institutional Animal Care and Use Committee approval. For Case Reports or Series, and Images in Endocrinology, consent forms, are required for the publication of information about patients, otherwise, appropriate ethical clearance has been obtained from the institutional review board. Articles and any other material published in the JAFES represent the work of the author(s) and should not be construed to reflect the opinions of the Editors or the Publisher. 